\title{
Rheumatic Heart Disease
}

National Cancer Institute

\section{Source}

National Cancer Institute. Rheumatic Heart Disease. NCI Thesaurus. Code C34882.

An autoinflammatory condition following an infection with Group A Beta Hemolytic

Streptococcus (GABHS), in which the heart is attacked by antibodies formed in reaction

to a recent GABHS infection. Chief anatomic changes of the valve include leaflet

thickening, commissural fusion, and shortening and thickening of the tendinous cords, all of which can result in valvular dysfunction. 